\title{
Practical use of sevelamer in chronic kidney disease patients on dialysis in People's Republic of China
}

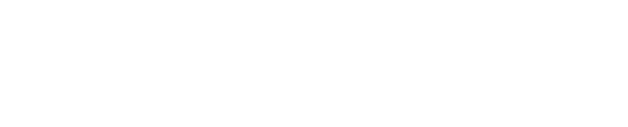

Lin Meng ${ }^{1,2}$

Bin Fu'

'Department of Nephrology, Second Affiliated Hospital to Tianjin University of Traditional Chinese Medicine, Tianjin, People's Republic of China; ${ }^{2}$ Tianjin University of Traditional Chinese Medicine, Tianjin, People's Republic of China
Correspondence: Bin Fu Second Affiliated Hospital to Tianjin University of Traditional Chinese Medicine, 86I ZhenLi Street, Tianjin, 300150 , People's Republic of China Tel +862260335393

Email fubin_nephrology@।63.com
Abstract: Hyperphosphatemia is a common complication of dialysis patients. Only $38.5 \%$ of Chinese dialysis patients met the Kidney Disease Outcomes Quality Initiative defined targets for serum phosphate. Sevelamer is a high molecular weight cationic hydrogel polymer that prevents absorption of dietary phosphate by binding it in the gastrointestinal tract. In Chinese trials, it was confirmed that sevelamer had better efficacy than calcium carbonate in terms of reducing the serum level of phosphorus and calcium-phosphate product. Sevelamer can also reduce the levels of lipid parameters and improve the micro-inflammatory state. When sevelamer was combined with other treatments, it elicited superior effects on calcium phosphorus metabolism, secondary hyperparathyroidism, and renal osteodystrophy. Combination treatment of sevelamer and traditional Chinese medicine has the unique advantage. However, sevelamer is associated with a high incidence of gastrointestinal adverse effects in Chinese patients. Although more effective, the practical use of sevelamer is not very common because it is expensive and not paid by medical insurance. This article provides a comprehensive review of the practical use of sevelamer in chronic kidney disease patients on dialysis in People's Republic of China.

Keywords: sevelamer, dialysis, People's Republic of China, phosphate binders, efficacy, safety

\section{Introduction}

In recent years, the amount of dialysis patients is rising gradually in People's Republic of China as the population ages and survival rates of this patient population improve. Hyperphosphatemia is a common complication of dialysis patients. The effect of restriction of dietary phosphorus is not ideal. Insufficient use of phosphorus binders was observed in People's Republic of China. Only 38.5\%, 39.6\%, and 26.6\% of Chinese dialysis patients met the Kidney Disease Outcomes Quality Initiative defined targets for serum phosphate, calcium, and intact parathyroid hormone (iPTH) levels in the Practice Patterns and Improvement Study of Bone Metabolism and Disease in patients with Chronic Kidney Disease (PPIS). ${ }^{1}$ The issues of hyperphosphatemia and mineral and bone disorder are prominent among Chinese patients receiving dialysis and need further attention. Non-calcium containing phosphorus binders were not available in many hemodialysis (HD) and peritoneal dialysis centers in People's Republic of China. Sevelamer for practical use was mainly distributed in tertiary hospitals. In addition, the practical use of carbonate sevelamer is less than hydrochloride sevelamer.

\section{Overview of sevelamer}

Sevelamer is a high molecular weight $(1,016 \mathrm{Da})$ cationic hydrogel polymer/resin that prevents absorption of dietary phosphate by binding it in the gastrointestinal (GI) tract., ${ }^{2,3}$ Sevelamer has sustained a clinically preferred safety profile over other calcium-based 
Table I The two salt forms of sevelamer

\begin{tabular}{lll}
\hline Forms & Sevelamer hydrochloride & Sevelamer carbonate \\
\hline Dosage form & Capsules & Tablets \\
Trade name & Renagel & Renvela \\
Approved for marketing by US Food and Drug Administration & In 1998 & In 2007 \\
First marketed in People's Republic of China & In 2010 & $\ln 2013$ \\
Chemical formula & $(\mathrm{C} 6 \mathrm{H}$ I2NClO)n & $(\mathrm{C} 3 \mathrm{H} 7 \mathrm{~N}) \mathrm{M}(\mathrm{C} 3 \mathrm{H} 5 \mathrm{CLO}) \mathrm{N}(\mathrm{CH} 2 \mathrm{O} 3) \mathrm{X}$ \\
\hline
\end{tabular}

or non-calcium containing binders because it is not absorbed, hence poses no risk of cation-induced toxicities. ${ }^{4}$ There are two salt forms in sevelamer, the hydrochloride form and the carbonate form (Table 1). Among the two available salt forms, the carbonate form of sevelamer is better tolerated in patients than the previous hydrochloride form, as the latter often exacerbates the tendency of patients with advanced kidney disease to metabolic acidosis. ${ }^{5,6}$ However, no study was performed to compare the effects of sevelamer hydrochloride with sevelamer carbonate in People's Republic of China. The non-absorbable nature of sevelamer has been confirmed. . $^{3,7}$ More than $99 \%$ of the drug was excreted in the feces over a 10 -day period in the majority of volunteers. ${ }^{3}$

\section{Therapeutic efficacy}

The efficacy of sevelamer in Chinese patients on dialysis has been confirmed in a number of trials (Table 2)..$^{8-15}$

\section{Monotherapy}

It has been proved that sevelamer had efficacy on reducing the serum level of phosphorus and calcium-phosphate product. However, the serum level of calcium and $\mathrm{PTH}$ of the patients remained steady in sevelamer treatment. Meanwhile, sevelamer can reduce the levels of blood lipid, attenuate the vascular calcification, and improve the microinflammatory state.

\section{Impact of sevelamer on calcium phosphorus metabolism}

A series of studies were designed to assess the efficacy of sevelamer on hyperphosphatemia in chronic kidney disease (CKD) patients on dialysis in People's Republic of China. ${ }^{8-15}$ Chen et $\mathrm{al}^{8}$ performed a randomized, double-blind, dose-titration study which compared sevelamer carbonate (starting dose $800 \mathrm{mg}$ three times daily) with placebo over 8 weeks' duration in Chinese CKD patients on HD. The mean serum phosphorus decreased significantly in patients treated with sevelamer carbonate (change $-0.69 \pm 0.64 \mathrm{mmol} / \mathrm{L}$ ) but remained persistently elevated with placebo (change $-0.64 \pm 0.57 \mathrm{mmol} / \mathrm{L})(P<0.0001)$.
Fang et $\mathrm{al}^{9}$ performed an open-labeled, self-control study, the data of which came from five large hospitals of three different cities (Beijing, Shanghai, and Guangzhou). Onehundred and thirty-eight patients with more than $1.78 \mathrm{mmol} / \mathrm{L}$ serum phosphorus after 2-week washout period comprised the study. By the end of 10-week sevelamer hydrochloride treatment, the serum level of phosphorus, calcium-phosphate product significantly decreased, while the adjusted serum level of calcium and serum iPTH remained steady. In another similar study ${ }^{11,21}$ maintenance hemodialysis (MHD) patients were treated with sevelamer carbonate for 16 weeks. By the end of treatment, compared with before treatment, the level of serum phosphorus and calcium-phosphorus product significantly decreased, while the serum levels of calcium and iPTH had no significant change.

In some controlled trials, it was confirmed that sevelamer had better efficacy than calcium carbonate in terms of reducing the serum level of phosphorus and calcium-phosphate product. $^{10,12,13}$ For example, Huang ${ }^{12}$ performed a 10 -week study in 62 Chinese patients undergoing MHD that evaluated the efficacy of sevelamer hydrochloride in treating hyperphosphatemia. The patients were randomly assigned to two groups, 31 cases in each group. The control group received calcium carbonate D treatment and the treatment group received sevelamer hydrochloride treatment. At week 10, the serum levels of phosphorus $(1.84 \pm 0.51 \mathrm{mmol} / \mathrm{L})$ and calciumphosphate product $\left(4.17 \pm 1.73 \mathrm{mmol}^{2} / \mathrm{L}^{2}\right)$ of the treatment group were lower than the control group $(2.19 \pm 0.35 \mathrm{mmol} / \mathrm{L}$ and $5.16 \pm 1.65 \mathrm{mmol}^{2} / \mathrm{L}^{2}$, respectively, $\left.P<0.05\right)$.

In the vast majority of research, the serum level of iPTH of the patients in sevelamer treatment remained steady. ${ }^{9,11,12}$ However, in the study of He et $\mathrm{al}^{13}$ short-term efficacy on four types of phosphate binders (calcium carbonate, sevelamer hydrochloride, lanthanum carbonate, and aluminum hydroxide) in MHD patients was evaluated. The results showed that iPTH decreased, but the decline of iPTH was not due to sevelamer. That was because high-flux dialyzers which can effectively remove $\mathrm{PTH}^{16}$ were used, and dialysis time of 12-14 hours per week was ample. In addition, the serum level of iPTH decreased in all four groups in this study. 


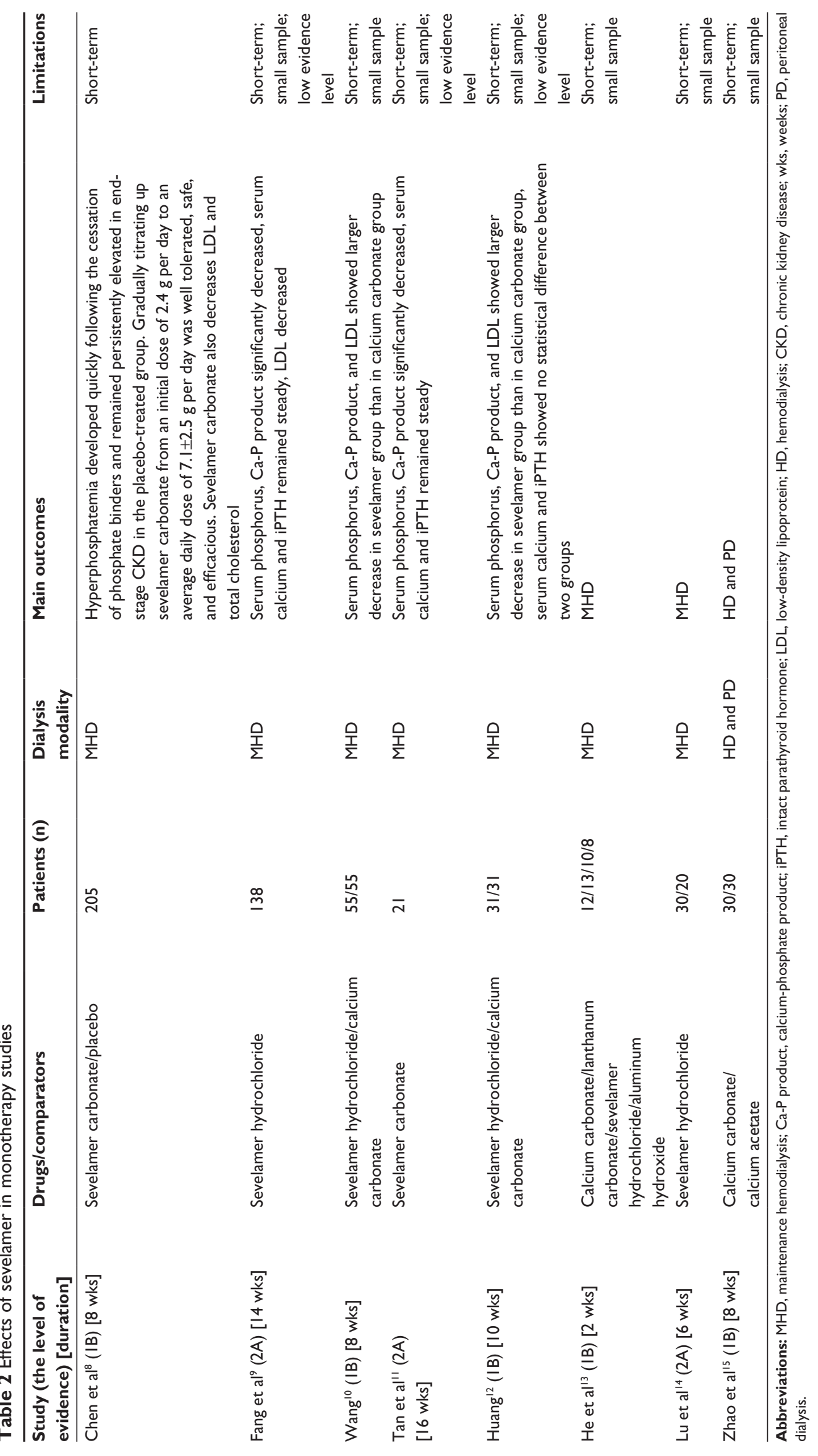


A review showed that sevelamer was at least as effective as calcium acetate and calcium carbonate at controlling serum phosphorus, calcium-phosphorus product in patients from other countries. ${ }^{17}$ However, as we saw from above trials, ${ }^{10,12,13}$ sevelamer had better efficacy than calcium carbonate in terms of reducing the serum level of phosphorus and calcium-phosphate product. Food consumption in Chinese individuals is characterized by a higher intake of whole grains, legumes, vegetables, and fruits. ${ }^{18}$ In this plant-based diet, phosphate is primarily stored in the form of phytate. ${ }^{19,20}$ Phytate phosphate is almost unavailable. However, the use of active vitamin D sterols increases the bioavailability of phytate phosphate, ${ }^{21-25}$ which can lead to an increase of serum phosphorus. Fortunately, sevelamer was effective at binding phytate phosphorus that is liberated by active vitamin D. ${ }^{26}$ Hence, sevelamer was more effective in People's Republic of China. Another reason is probably that the body size of Chinese are generally smaller, so the drug dosage is relatively enough.

\section{Impact of sevelamer on the blood lipid}

Sevelamer can reduce the levels of lipid parameters. It has been proven that sevelamer can reduce low-density lipoprotein (LDL) in Chinese patients on dialysis. ${ }^{8-10,12,14}$ When compared with placebo, sevelamer carbonate treatment resulted in statistically significant greater mean reductions from baseline in serum total $(-17.1$ versus $-3.3 \%)$ and LDL cholesterol $(-33.5$ versus $-7.6 \%)(P<0.0001$ for both). ${ }^{8}$ Compared with calcium-containing phosphorus binders, sevelamer was more effective in reducing LDL (Table 2). ${ }^{10,12}$ In the study of Lu et al ${ }^{14}$ not only LDL but also total triglycerides and total cholesterol reduced under the sevelamer treatment, while the serum level of high-density lipoprotein had no significant change. Total cholesterol's reduction was also found in treatment with sevelamer hydrochloride in Germans and Dutch. ${ }^{27,28}$ Sevelamer has been shown to bind bile acids. ${ }^{29}$ Bile acid binding by ion exchange resins is a well-established method of lowering serum cholesterol. ${ }^{8}$

\section{Impact of sevelamer on the micro- inflammatory state}

$\mathrm{Lu}$ et $\mathrm{al}^{14}$ conducted a study to explore sevelamer hydrochloride's impact on the micro-inflammatory state. MHD patients with diabetic nephropathy were given sevelamer (800 $\mathrm{mg}$ three times a day with meals for 8 weeks), as a result, the serum levels of inflammatory marker CRP, IL-6, and TNF- $\alpha$ reduced.
Thirty-four MHD patients comprised the controlled study conducted by Deng et al. ${ }^{30}$ All patients received Caltrate D (900 mg qd [daily]) treatment at baseline. The treatment group received sevelamer hydrochloride $(800 \mathrm{mg}$ tid [three times a day]) and the control group received calcitriol $(0.25 \mu \mathrm{g} \mathrm{qd})$. After 12 weeks, there were more reductions in CRP, IL-6 concentration in the study group compared to the control group. The results of the study suggested that sevelamer hydrochloride was superior to calcitriol in improving the micro-inflammatory state.

\section{Impact of sevelamer on cardiovascular disease of dialysis patients}

Zhao et al ${ }^{15}$ measured serum FGF23, fetuin-A and high-sensitivity-CRP in 60 dialysis patients who completed an 8-week, controlled, randomized study. Thirty patients received sevelamer carbonate and 27 patients received calcium acetate. After 8-week treatment, the serum highsensitivity-CRP had a lower change in sevelamer carbonate group than in calcium acetate group $[(-3.28 \pm 1.23)$ versus $(-0.03 \pm 0.21) \mathrm{mg} / \mathrm{L}$, respectively, $P<0.05]$. The serum fetuin-A had a higher change in sevelamer carbonate group than in calcium acetate group $[(0.09 \pm 0.04)$ versus $(0.0053 \pm 0.16) \mathrm{g} / \mathrm{L}$, respectively, $P<0.05]$. The changes of FGF-23 are $-0.17 \pm 7.64 \mathrm{ng} / \mathrm{L}$ and $0.017 \pm 12.06 \mathrm{ng} / \mathrm{L}$, respectively, $P>0.05$, without statistical significance. CRP predicted all-cause and cardiovascular mortality in HD patients. ${ }^{31}$ Fetuins account for roughly half of the capacity of serum to inhibit salt precipitation. ${ }^{32}$ Therefore, the results of the study indicated that sevelamer can attenuate the vascular calcification and improve cardiovascular outcomes through the reduction of CRP and the increase of fetuin-A. Similarly, another study reported slower progression of coronary, aortic, and heart valve calcification with use of sevelamer in comparison to calcium acetate by using the Agatston scoring system in European and American MHD patients (seven in the US, seven in Germany, and one in Austria). ${ }^{33}$ In contrast, a study reported there was no difference in coronary artery calcification progression between the calcium and sevelamer groups in Brazil. ${ }^{34}$ Meta-analysis also showed existing data were insufficient to conclude a differential impact of any phosphate binder on cardiovascular mortality. ${ }^{35}$

\section{Combination therapy}

Sevelamer has been assessed in several trials when combined with other treatments such as traditional Chinese medicine, active vitamin D pulse therapy, or different dialysis model in Chinese patients on dialysis. 


\section{Combination treatment of sevelamer and traditional Chinese medicine}

Cuttlebone is a traditional Chinese medicine that lowers serum phosphate ${ }^{36-38}$ and its major component is calcium carbonate $(87.3 \% \sim 91.8 \%)$. In the study conducted by Zhu et a $1^{39} 90$ cases of uremia in HD were randomly divided into the cuttlebone treatment group (group 1), the cuttlebone combined with sevelamer treatment group (group 2) and the sevelamer treatment group (group 3). After treatment for 8 weeks, serum phosphate decreased in all groups, but its reduction is more obvious in group 2 and group 3 than in group 1. Serum phosphate was reduced to normal in $47.2 \%$ of patients through the combination treatment of sevelamer and cuttlebone, moreover, calcium-phosphate product, LDL, and $\mathrm{PTH}$ were lowered.

Cuttlebone is one of the traditional Chinese medicines whose major component is calcium carbonate. Cuttlebone had efficacy on reducing the serum level of phosphorus, with little effect on serum calcium. ${ }^{40,41}$ As a phosphate binder, cuttlebone is rich in natural calcium. Perhaps calcium granules in cuttlebone are big, thus not easily absorbed by the body when taken orally, so cuttlebone had little effect on serum calcium. Besides being rich in calcium carbonate, cuttlebone contains elements such as $\mathrm{Mg}$ and $\mathrm{Fe}$, which also blocked phosphorus absorption. Furthermore, the side effect of constipation in cuttlebone group was less than in calcium carbonate group. ${ }^{42}$ Therefore, the combination treatment of sevelamer and traditional Chinese medicine has great prospects for development.

\section{Effect on secondary hyperparathyroidism (SHPT)}

SHPT is one of the major complications of uremia. Pulse doses of active vitamin D was usually used for SHPT treatment, but it is frequently accompanied by hypercalcemia, hyperphosphatemia, and metastatic calcification. The combination treatment of sevelamer, pulse doses of active vitamin $\mathrm{D}$, and other treatments can significantly decrease iPTH for SHPT patients, with little occurrence of hypercalcemia, hyperphosphatemia, and metastatic calcification. ${ }^{43-46}$

In the study by Zhao et al ${ }^{43} 34 \mathrm{MHD}$ patients, with iPTH levels of 33-55 pmol/L, were treated with calcitriol $(1.0 \mu \mathrm{g}$ twice a week) pulse therapy. Eighteen of the patients (the study group) were treated with oral Renagel ( $800 \mathrm{mg}$, tid), and 16 of the patients (the control group) were treated with calcium carbonate $900 \mathrm{mg} / \mathrm{d}$. Serum phosphate and iPTH levels 2 months after treatment in the study group were significantly lower than those before treatment. The levels of serum phosphate in the study group were significantly lower than those in the control group $(P<0.05)$. Sevelamer was more effective in improving the hyperphosphatemia in MHD patients with SHPT in calcitriol pulse therapy. Consistent with the above study, in the study by Ding et $\mathrm{al}^{44}$ even in severe SHPT (iPTH $>1,000 \mathrm{pg} / \mathrm{mL}$ ) patients, the combination therapy was still obviously effective, meanwhile, serum levels of uric acid also lowered.

Liu et $\mathrm{al}^{45}$ conducted a 6 -month trial to observe the effect of the combination treatment of low calcium dialysate $(1.25 \mathrm{mmol} / \mathrm{L})$, active vitamin $\mathrm{D}$, and sevelamer hydrochloride on SHPT in chronic renal failure patients with MHD. As compared with those before the treatment, serum phosphate, calcium-phosphorus product, and iPTH decreased after treatment for 3 months, and decreased further after the treatment for 6 months. Serum iPTH varied dramatically. In the 30 cases who were recruited before the treatment, after the treatment for 3 months and for 6 months, serum iPTH was $650.80 \pm 119.40 \mathrm{pg} / \mathrm{mL}, 376.10 \pm 117.40 \mathrm{pg} / \mathrm{mL}$, and $211.90 \pm 109.40 \mathrm{pg} / \mathrm{mL}$, respectively. This combination therapy prevents hypercalcemia and metastatic calcification, and it is effective in renal osteopathy of high transport type.

\section{Effect on renal osteodystrophy}

Renal osteodystrophy is a highly prevalent complication in patients on dialysis, causing pain and significant fractureassociated morbidity and mortality. The usual management of renal osteodystrophy involves the use of active vitamin D and phosphate binders, even surgical removal of parathyroid glands, but the clinical curative effect is not very satisfactory. The combination treatment of sevelamer and other treatment methods has obvious curative effects on renal osteodystrophy. ${ }^{47-49}$

$\mathrm{Xu}$ et $\mathrm{al}^{47}$ conducted a 3-month trial to investigate the effect of the combination treatment of sevelamer hydrochloride and hemodiafiltration on renal osteodystrophy. A total of 69 cases with renal osteodystrophy were divided into three groups according to the treatment, 23 cases in each group, the control group A received sevelamer hydrochloride oral treatment, the control group $B$ received hemodiafiltration and the treatment group received sevelamer hydrochloride and hemodiafiltration. The efficacy and common biochemical parameters before and after treatment of the three groups were compared. The total effective rate of the treatment group was significantly higher than that of the control group $\mathrm{A}$ and control group $\mathrm{B}$, with statistical significance $(P<0.05)$.The serum phosphorus, calcium-phosphorus product, ALP, $\beta-\mathrm{MG}$, and PTH were lower than before treatment, 
with statistical significance $(P<0.05)$. Serum phosphorus, calcium-phosphorus product, ALP, $\beta-\mathrm{MG}$, and PTH were lower in the treatment group than the control group A and control group $\mathrm{B}$, with statistical significance $(P<0.05)$. The effect of the combination treatment of sevelamer and hemodiafiltration on renal osteodystrophy is obvious.

A study was performed to evaluate the effect of triple therapy on renal osteodystrophy. Forty-four cases with renal osteodystrophy were divided into the study group and the control group. The study group received triple therapy (the combination treatment of hydrochloride sevelamer and low calcium dialysate and calcitriol pulse therapy) and the control group received calcitriol ( $2 \mu \mathrm{g}$, qod [every other day]) and aluminum hydroxide gel, which was replaced by calcium carbonate when calcium-phosphorus product was less than $60 \mathrm{mg}^{2} / \mathrm{dL}^{2}$. After treatment for 3 months, calciumphosphorus product and iPTH were significantly lower in the study group than the control group. Besides, the serum levels of IL- 6 and IL-12 declined in the two groups, and the degree is bigger in the study group, which showed clearly that triple therapy had anti-inflammatory effects on renal osteodystrophy. ${ }^{48}$ A similar result was found by Liu et al. ${ }^{49}$ Calcitriol can inhibit the synthesis and secretion of PTH.$^{50}$ It has been the main treatment for renal osteodystrophy, but it often causes complications such as hypercalcemia, the rise of calcium-phosphorus product so that the treatment has to be discontinued. Sevelamer can effectively decrease serum phosphorus and rarely cause a rise in serum calcium and calcium-phosphorus product. ${ }^{46}$ Thus, as an ideal drug combination, sevelamer has a unique advantage on renal osteodystrophy, which has been proven by above studies. ${ }^{47-49}$

\section{Safety and tolerability}

In the studies reviewed above, the side effects of sevelamer often occurred, but there were no serious adverse events. The common side effects reported in People's Republic of China were mild-to-moderate GI disorders, such as constipation, flatulence, abdominal distension, dyspepsia, vomiting, nausea, etc, the most commonly observed was constipation. While, in the study of patients $(n=200)$ from the US, Germany, and Austria, the most common adverse events after 52 weeks of treatment with sevelamer were vomiting (22\%), nausea (20\%), diarrhea (19\%), and dyspepsia (16\%). ${ }^{4}$ In the study conducted by Fang et $\mathrm{al}^{9}$ the incidence rate of GI symptoms of sevelamer hydrochloride was $68.1 \%$, higher than in European and American people, while the incidence was $57.4 \%$ in Japanese people, ${ }^{51}$ which suggests that it was apt to occur in Asian patients. GI symptoms disappeared after proper treatment. Chen et $\mathrm{al}^{8}$ reported that adverse events experienced by patients in the sevelamer carbonate and placebo treatment groups were similar and consistent with their underlying renal disease, with the exception of constipation, which was more frequent in the sevelamer carbonate group.

He et $\mathrm{al}^{13}$ reported that the incidence of side effects in four groups (the calcium carbonate group, the sevelamer hydrochloride group, the lanthanum carbonate group, and the aluminum hydroxide group) did not show statistically significant difference $(P>0.05)$. In the study of Huang, ${ }^{12}$ the incidence of side effects in the sevelamer hydrochloride group $(77.4 \%)$ was higher than in the calcium carbonate group $(35.5 \%)$. However, it is interesting that when combined with calcitriol pulse therapy, the incidence of side effects in the sevelamer hydrochloride group $(30.0 \%)$ is lower than in the calcium carbonate group $(85.0 \%) .{ }^{44}$ Other side effects such as hypercalcemia, hypocalcemia were also reported during the clinical studies in People's Republic of China, 9,12,44 while pruritus, rash was not reported in Chinese trails. Sevelamer was well tolerated. In Chen et al's study, sevelamer carbonate was well tolerated with $96 \%$ adherence compared with $97 \%$ adherence in the placebo arm. ${ }^{8}$ Studies with small samples did not provide enough information on safety and tolerability of the drug, so evidence-based and high-quality medicine data are required in Chinese patients.

\section{Patient focused perspectives}

Sevelamer is a novel option for dialysis patients with hypercalcemia. Sevelamer is more expensive than the commonly used phosphate binders for most patients and it is not paid by medical insurance, so its awareness was low in low-income groups. Meanwhile, patients dislike its disadvantages such as large dose burden, the strict direction to be taken three times daily with meals, and GI side effects.

\section{Conclusion}

Sevelamer is effective in reducing the serum level of phosphorus, calcium-phosphate product and lipid levels without increasing serum calcium levels in Chinese dialysis patients both as a monotherapy and as an agent combined with other treatment.

When sevelamer was combined with other treatments, especially active vitamin D pulse therapy, it elicited superior effects on calcium phosphorus metabolism, SHPT, and renal osteodystrophy in Chinese patients on dialysis. Combination treatment of sevelamer and traditional Chinese medicine has the unique advantage. 
The population studied are mainly patients on HD and came from developed cities or provincial capitals. At present, most of the sevelamer studies in Chinese patients are single-center, short-term, and open-labeled. Therefore, more well-designed, large-scale, and long-term trials are needed to successfully assess the benefits and harms of sevelamer in CKD patients on dialysis in People's Republic of China.

\section{Acknowledgment}

We thank Hongfei Sui and Bo Zhang for assistance with preparation of this study.

\section{Disclosure}

The authors report no conflicts of interest in this work.

\section{References}

1. Kong X, Zhang L, Zhang L, et al. Mineral and bone disorder in Chinese dialysis patients: a multicenter study. BMC Nephrol. 2012; 9(13): 116 .

2. Burke SK, Amin NS, Incerti C, Plone MA, Lee JW. Sevelamer hydrochloride (Renagel), a phosphate-binding polymer, does not alter the pharmacokinetics of two commonly used antihypertensives in healthy volunteers. J Clin Pharmacol. 2001;41(2):199-205.

3. Plone MA, Petersen JS, Rosenbaum DP, Burke SK. Sevelamer, a phosphate-binding polymer, is a non-absorbed compound. Clin Pharmacokinet. 2002;41(7):517-523.

4. Chertow GM, Burke SK, Raggi P; Treat to Goal Working Group. Sevelamer attenuates the progression of coronary and aortic calcification in hemodialysis patients. Kidney Int. 2002;62(1):245-252.

5. Oka Y, Miyazaki M, Matsuda H, et al. Sevelamer hydrochloride dosedependent increase in prevalence of severe acidosis in hemodialysis patients: analysis of nationwide statistical survey in Japan. Ther Apher Dial. 2014;18(1):37-43.

6. Pai AB, Shepler BM. Comparison of sevelamer hydrochloride and sevelamer carbonate: risk of metabolic acidosis and clinical implications. Pharmacotherapy. 2009;29(5):554-561.

7. Burke SK, Slatopolsky EA, Goldberg DI. Renagel, a novel calciumand aluminium-free phosphate binder, inhibits phosphate absorption in normal volunteers. Nephrol Dial Transplant. 1997;12(8):1640-1644.

8. Chen N, Wu X, Ding X, et al. Sevelamer carbonate lowers serum phosphorus effectively in haemodialysis patients: a randomized, double-blind, placebo-controlled, dose-titration study. Nephrol Dial Transplant. 2014;29(1):152-160.

9. Fang Y, Ding XQ, Zou JZ, et al. Short-term efficacy of sevelamer hydrochloride on hyperphosphatemia in patients undergoing maintenance hemodialysis. Chinese Journal of Nephrology. 2012;28(3):183-188.

10. Wang CY. Short-term efficacy analysis of sevelamer hydrochloride on hyperphosphatemia in patients undergoing maintenance hemodialysis. China Heath Care Nutrition. 2013;23(1):301-302.

11. Tan HZ, Song XX, Liu CX. Efficacy of sevelamer carbonate on hyperphosphatemia patients undergoing maintenance hemodialysis. Journal of Xinjiang Medical University. 2013;38(7):984-978.

12. Huang ZY. Treatment of hyperphosphatemia with sevelamer hydrochloride in renal disease patients with MHD. Contemporary Medicine. 2014;20(23):68-69.

13. He YJ, Li Y, Ke JT, et al. Short-term efficacy and Safety Observation of 4 different types phosphate binders. Chin J Blood Purif. 2013;12(11): 632-634.

14. Lu L, Hu FQ, Gong JH, et al. Sevelamer hydrochloride impact on the microinflammatory state in diabetic kidney disease patients with MHD. Clin Nephrol. 2014;14(6):376.
15. Zhao Y, Cheng H, Li SL, et al. Efficacy of sevelamer carbonate on C-Reactive protein, fetuin-A and FGF-23 in end stage renal disease patients. Chinese Journal of Integrated Traditional and Western Nephrology. 2014;15(9):808-811.

16. Rui HR, Zhao YJ, Lin WM. High-flux dialyzers impact on serum phosphorus and PTH of patients undergoing maintenance hemodialysis. Journal of the 4th Military Medical University. 2005;26(7):664.

17. Goldsmith DR, Scott LJ, Cvetkovic' RS, Plosker GL. Sevelamer Hydrochloride: A Review of its Use for Hyperphosphataemia in Patients with End-Stage Renal Disease on Haemodialysis. Drugs. 2008;68(1): 85-104.

18. Hu FB. Dietary pattern analysis: a new direction in nutritional epidemiology. Curr Opin Lipidol. 2002;13(1):3-9.

19. Uribarri J. Phosphorus homeostasis in normal health and in chronic kidney disease patients with special emphasis on dietary phosphorus intake. Semin Dial. 2007;20(4):295-301.

20. Ravindran V, Bryden WL, Kornegay ET. Phytates: occurrence, bioavailability, and implications in poultry nutrition. Avian Poultry Biol Rev. 1995;6(2):125-143.

21. Lee DB, Walling MW, Brautbar N. Intestinal phosphate absorption: influence of vitamin D and non-vitamin D factors. Am J Physiol. 1986;250(3 Pt 1):G369-G373.

22. Mohammed A, Gibney MJ, Taylor TG. The effects of dietary levels of inorganic phosphorus, calcium and cholecalciferol on the digestibility of phytate-P by the chick. Br J Nutr. 1991;66(2):251-259.

23. Peterlik M, Wasserman RH. Regulation by vitamin D of intestinal phosphate absorption. Horm Metab Res. 1980;12(5):216-219.

24. Pointillart A, Fontaine N, Thomasset M. Effects of vitamin D on calcium regulation in vitamin-D-deficient pigs given a phytate-phosphorus diet. Br J Nutr. 1986;56(3):661-669.

25. Snow JL, Baker DH, Parsons CM. Phytase, citric acid, and 1 alphahydroxycholecalciferol improve phytate phosphorus utilization in chicks fed a corn-soybean meal diet. Poult Sci. 2004;83(7):1187-1192.

26. Bobeck EA, Meyer KM, Helvig C, Petkovich M, Cook ME. Sevelamer hydrochloride binds phosphate released from phytate in chicks fed 1 $\alpha$-hydroxy cholecalciferol. J Ren Nutr. 2013;23(1):21-27.

27. Brandenburg VM, Schlieper G, Heussen N, et al. Serological cardiovascular and mortality risk predictors in dialysis patients receiving sevelamer: a prospective study. Nephrol Dial Transplant. 2010; 25(8):2672-2679

28. Noordzij M, Korevaar JC, Boeschoten EW, et al. The Kidney Disease Outcomes Quality Initiative(K/DOQI) Guideline for Bone Metabolism and Disease in CKD: association with mortality in dialysis patients. Am J Kidney Dis. 2005;46(5):925-932.

29. Braunlin W, Zhorov E, Guo A, et al. Bile acid binding to sevelamer HCl. Kidney Int. 2002;62(2):611-619.

30. Deng ZP, Luo FZ, Li LB, et al. Sevelamer hydrochloride (Renagel) impact on the microinflammatory state of MHD patients. Medical Information. 2012;25(10):107.

31. Yeun JY, Levine RA, Mantadilok V, Kaysen GA. C-Reactive protein predicts all-cause and cardiovascular mortality in hemodialysis patients. Am J Kidney Dis. 2000;35(3):469-476.

32. Schinke T, Amendt C, Trindl A, Pöschke O, Müller-Esterl W, JahnenDechent W. The serum protein alpha2-HS glycoprotein/fetuin inhibits apatite formation in vitro and in mineralizing calvaria cells. A possible role in mineralization and calcium homeostasis. J Biol Chem. 1996; 271(34):20789-20796.

33. Chertow GM, Burke SK, Raggi P; Treat to Goal Working Group. Sevelamer attenuates the progression of coronary and aortic calcification in hemodialysis patients. Kidney Int. 2002;62(1):245-252.

34. Barreto DV, Barreto Fde C, de Carvalho AB, et al. Phosphate binder impact on bone remodeling and coronary calcification-Results from the BRiC Study. Nephron Clin Pract. 2008;110(4):c273-c283.

35. Navaneethan SD, Palmer SC, Craig JC, Elder GJ, Strippoli GF. Benefits and harms of phosphate binders in CKD: a systematic review of randomized controlled trials. Am J Kidney Dis. 2009;54(4): 619-637. 
36. Yi HC, Tang LH, Zhang XP. Experimental Study on Bone Defect Treated by Combined Autologous Bona Marrow Transplantation, Cuttebone, and Sodium Hyaluronate. Chinese Journal of Integrated Traditional and Western Medicine. 2011;31(8):1122-1123.

37. Shen YF, Shen JG, Zhu SD. Advance in pharmacological action of Cuttlebone, a traditional Chinese medicine. China Pharmaceuticals. 2010;19(10):87-89.

38. Wu CW, Chi CF, He GY, et al. Determination of 8 trace elements in mantle muscle and cuttlebone of sepiella maindroni by ICP-MS using microwave digestion for sample preparation. Spectroscopy and Spectral Analysis. 2009;29(12):3395-3398.

39. Zhu QX, Li YD. Effect Analysis of cuttlebone combined with sevelamer on hyperphosphatemia patients with uremia undergoing maintenance hemodialysis. Contemporary Medicine. 2013;19(36):103-105.

40. Li JQ, Li XF, Zhou WW, et al. Clinical study on Effects of sevelamer on calcium phosphorus metabolism in hemodialysis uremic patients. Chinese Journal of Integrated Traditional and Western Nephrology. 2012;13(3):246-247.

41. Liu LS, Hu G. Study on effect of cuttlebone on serum phosphorus and PTH in hemodialysis uremic patients. Practical Clinical Journal of Integrated Traditional Chinese and Western Medicine. 2005;5(5):5-6.

42. Guo YX. Study on treating hyperphosphatemia with cuttlebone in peritoneal dialysis patients. Zhejiang Clinical Medical Journal. 2008;10(9):1236-1237.

43. Zhao J, Chen XL, Zhang JF, et al. Effect of sevelamer hydrochloride on serum phosphorus in uremia patients with administration of calcitrio pulse therapy on SHPT. Hainan Medical Journal. 2012;23(18):37-38.
44. Ding Y. Combination therapy on severe SHPT patients on dialysis with sevelamer hydrochloride and low dose calcitrio pulse therapy. Medical Information. 2014;27(6):68-69.

45. Liu WH, Hu WB, Wang XR. Clinical Observation on treating secondary hyperparathyroidism in dialysis patients with low calcium dialysate combined with active vitamin D and sevelamer hydrochloride. Chin J Blood Purif. 2014;7(13):493-496.

46. Du Y, Li M, Li J, et al. Effect of calcitrio combined with sevelamer hydrochloride on serum PTH in chronic renal failure patients. Chinese Journal of Clinicians (electronic edition). 2011;05(2):505-507.

47. Xu MJ, Shao JY. Effect of sevelamer hydrochloride combined with hemodiafiltration on renal osteodystrophy. China Modern Doctor. 2014;52(18):45-47.

48. Ye Q, Zhang JW. Effect of triple therapy on renal osteodystrophy and on the serum levels of IL-6 and IL-12. Guangdong Medical Journal. 2012;33(15):2248-2249.

49. Liu ZG, Ren LY, Ren SE. Study on treating renal osteodystrophy with low calcium dialysate combined with sevelamer hydrochloride and calcitrio pulse therapy. China Prac Med. 2014;9(25):30-31.

50. Hernandez JD, Wesseling K, Salusky IB. Role of parathyroid hormone and therapy with active vitamin D sterols in renal osteodystrophy. Semin Dial. 2005;18(4):290-295.

51. Ogata H, Koiwa F, Shishido K, Kinugasa E. Combination therapy with sevelamer hydrochloride and calcium carbonate in Japanese patients with long-term hemodialysis: alternative approach for optimal mineral management. Ther Apher Dial. 2005;9(1):11-15.
Therapeutics and Clinical Risk Management

\section{Publish your work in this journal}

Therapeutics and Clinical Risk Management is an international, peerreviewed journal of clinical therapeutics and risk management, focusing on concise rapid reporting of clinical studies in all therapeutic areas outcomes, safety, and programs for the effective, safe, and sustained use of medicines. This journal is indexed on PubMed Central, CAS,

\section{Dovepress}

EMBase, Scopus and the Elsevier Bibliographic databases. The manuscript management system is completely online and includes a very quick and fair peer-review system, which is all easy to use. Visit http://www.dovepress.com/testimonials.php to read real quotes from published authors. 